\title{
Detection and Comparison of Objects Using Two Dimensional Geometric Moment Invariants
}

\author{
Y. Gangadhar, D. Lakshmi Srinivasulu, and V. S. Giridhar Akula, Senior Member, IACSIT
}

\begin{abstract}
In this paper, we present the concept of Moment Invariants. Objects can be detected with the help of Moment Invariants. Different Moment Invariants are discussed in this paper. The pitfalls of Fourier-Mellin Invariants and Legendre Moment Invariants are discussed. Two Dimensional Moments of $\mathrm{N} \times \mathrm{N}$ images are sampled and proved that $2 \mathrm{D}$ Geometrical Moment Invariants are far better than the other Invariants in terms of the object detection.
\end{abstract}

Index Terms-Image function, algebraic invariants, polar coordinates, affine groups, information redundancy, fourier-mellin invariants, legendre moment invariants, counter-based shape descriptors, geometric moment invariants.

\section{INTRODUCTION}

Moment Invariants are commonly used for identification and inspection of shapes of images. Moment Invariants plays a vital role in many applications of computer vision and pattern recognition. The basic idea of Moment Invariants is to describe the objects by a group of features which provide discrimination power to identify objects from different groups. The 2D Moment Invariants were firstly introduced by $\mathrm{Hu}$ [1] in 1962. Who used the results of theory of algebraic invariants and derived seven known invariants to rotation of 2D objects. Dudani [2] and Belkasim [3] explained their work on applications of aircraft silhouette recognition. Li [4] and Wong [5] exploded the invariants order to fine and fire respectively. Flusser and Suk[6] employed moment invariants in template matching and recognition of satellite images. Wang [7] proposed illumination invariants suitable for feature classification. Resis [8] revised some of the geometrical proofs of $\mathrm{Hu}$. Teague [9] proposed the use of orthogonal moments based on the history of orthogonal polynomials. Different types of Image Moments, like Geometrical Moments, Legendre Moments, Zernike Moments, Fourier- Mellin Moments, Pseudo-Zernike Moments, Complex Moments were developed based on the capability of image description, noise sensitivity etc., Wallin [10] discussed an algorithm for the formation of moment invariants of any order. Flusser [11] discussed the independence of Two Dimensional Rotational Moments via Complex Moments. He also constructed 2D moment invariants basis and its independence and completeness. Van

Manuscript received May 10, 2012; revised June 19, 2012

Y. Gangadhar is with the Dept of CSE, Avanthi's Scientific Technological \& Research Academy, Hyderabad-501512, India (e-mail: ygdhar@gmail.com).

D. Lakshmi Srinivasulu is with the Dept of CSE, Santhiram Engineering College, Nandyal-518501, India (e-mail: dlsrinivas@gmail.com).

V. S. Giridhar Akula is with the Avanthi's Scientific Technological \& Research Academy, Hyderabad-501512, India (e-mail: seshagiridhar.a@gmail.com).
Gool [12] introduced affine-photo metric invariants of gray level and color images. Flusser and Suk [13] derived invariants to convolution which an arbitrary PSF. But these descriptions were invariants to translation only.

\section{THE CONCEPT OF MOMENT INVARIANTS}

Moment invariants were first introduced by the $\mathrm{Hu}$. By image functions we understand any real function $f(x, y) \in$ $\mathrm{Li}$ having a bounded support and a non-zero integral. Translation and scale variance of a dimension moment invariants are easy to be eliminated. Let $m_{p_{1} \ldots p_{n}}$ represent the n-dimension $\left(p_{1}+\cdots+p_{n}\right)^{\text {th }}$ order moment of a piecewise continuous density function $h\left(x_{1}, \ldots, x_{n}\right)$, it can be defined as

$$
m_{p_{1} \ldots p_{n}=\int \ldots . \int_{\infty} x_{1}^{p_{1}} \ldots \ldots x_{n}^{p_{n}} h\left(x_{1}, \ldots ., x_{n}\right) d x_{1}, \ldots ., d x_{n}}
$$

The scale variants can be eliminated based on the concept of algebraic invariants [14] as follows.

$$
\eta_{p_{1} \ldots p_{n}}=\frac{\mu_{p_{1} \ldots p_{n}}}{\mu_{0 \ldots 0}\left(p_{1}+\cdots+p_{n}\right) / n+1}
$$

\section{A. Expression for Moment-Based Rotation Invariants}

Rotation invariant is achieved by using Zernike's moment invariants, wavelet moment invariants and Li's moment invariants.

Let $f(x, y)$ represent a two dimensional binary image object in the $(x, y)$ coordinate and Let $f(r, \theta)$ be its corresponding polar coordinate. Then the relationship between $f(x, y)$ and $f(r, \theta)$ is specified as

$$
\begin{aligned}
& x=r \cos (\theta), \\
& y=r \sin (\theta) .
\end{aligned}
$$

To derive rotation invariant moments, the following general expression is used

$$
f_{p q}=\iint f(r, \theta) g_{p}(r) e^{j q \theta} r d r d \theta
$$

where $f_{p q}$ is the $p q$ order moment, $g_{p}(r)$ is a function of radial variable $r$, and $P$ and $q$ are integer parameters.

\section{FOURIER-MELLIN INVARIANTS}

The optical research community was introduced the Fourier-Mellin transform in 1970s. These transforms are used now-a-days in Digital Image and Signal Processing. The main concept of Fourier-Mellin transform is with the study of similarity transformations. Ghorbel [15] work focused on the Fourier transform defined on 2D and 3D parameterization. Tursci [16] explained some Fourier transforms for sub groups 
of the affine group.

Let $f$ denote a function representing a gray level image defined over a compact set of $\mathbb{R}^{2}$. The standard Fourier-Mellin Transform of $f$ is given by

$$
\begin{gathered}
\forall(k, v) \in \mathbb{Z} \times \mathbb{R}, \mu_{f}(k, v)= \\
\frac{1}{2 \pi} \int_{0}^{\infty} \int_{0}^{2 \pi} f(r, \theta) r^{-i r} e^{-i k \theta} d \theta \frac{d r}{r}
\end{gathered}
$$

$f$ Is assumed to be Summable over $\mathbb{R}_{+}^{*} \times S^{1}$ under the measure $\frac{d r}{r}$, ie

$$
\begin{gathered}
\int_{0}^{\infty} \int_{0}^{2 \pi}\left|f(r, \theta) r^{-i r} e^{-i k \theta}\right| d \theta \frac{d r}{r}= \\
\int_{0}^{\infty} \int_{0}^{2 \pi} \frac{1}{r} f(r, \theta) d \theta d r \quad<\infty
\end{gathered}
$$

\section{LEGENDRE MOMENTS}

Legendre moments were introduced by Teague [17]. Tel and chain [18] stated that orthogonal Legendre moments can be used to represent an image with a minimum amount of information redundancy.

The two dimensional Legendre moments of order $(p+q)$ for image intensity function $f(x, y)$ are defined as

$$
L_{p q}=\frac{(2 p+1)(2 q+1)}{4} \int_{-1}^{1} \int_{-1}^{1} p_{p}(x) p_{q}(y) f(x, y) d x d y
$$

where $p_{p}(x)$ is the $p$ th order Legendre polynomial Legendre descriptors of the third and fourth order are used to test the invariance.

Third order descriptions are

Fourth order descriptions are

$$
\begin{aligned}
& \phi_{1=\frac{35}{8} \eta_{3,0}} \\
& \phi_{2=\frac{45}{8} \eta_{2,1}} \\
& \phi_{3=\frac{45}{8} \eta_{1,2}} \\
& \phi_{4=\frac{35}{8} \eta_{0,3}}
\end{aligned}
$$

$$
\begin{gathered}
\phi_{5}=\frac{9}{4}\left[\frac{35}{8} \eta_{4,0}-\frac{30}{8} \eta_{2,0}+\frac{3}{8} \eta_{0,0}\right] \\
\phi_{6=\frac{105}{8} \eta_{3,1}}=\frac{25}{4}\left[\frac{9}{4} \eta_{2,2}-\frac{3}{4} \eta_{2,0}-\frac{3}{4} \eta_{0,2}+\frac{1}{4} \eta_{0,0}\right] \\
\phi_{8=\frac{105}{8} \eta_{1,3}} \\
\phi_{9}=\frac{9}{4}\left[\frac{35}{8} \eta_{0,4}-\frac{30}{8} \eta_{0,2}+\frac{3}{8} \eta_{0,0}\right]
\end{gathered}
$$

\section{PROPOSED WORK}

The proposed work in this paper used geometric moment invariants and formed to be superior compare Fourier-Mellin invariants and Legendre moments.

\section{A. Geometric Moment Invariants}

The Geometric Moment Invariants produce a group of features vectors that are invariants under shifting, rotation and scaling. Regular moment invariants are most popular counter-based shape descriptions derived by Hu. Geometric moment invariants were first introduced by $\mathrm{Hu}$, which are derived from the theory of algebraic invariants.
Geometric moment were successfully applied on the alphabet A shown in different shapes. The alphabet image A is used to get the range of invariant. The comparison of invariants feature vectors are shown in the table.

Two dimensional moments of a digitally sampled

$N \times N$ Image has gray function $f(x, y), \quad(x, y=$ $0 \ldots . . N-1)$

And is given by

$$
m_{p q}=\sum_{x=0}^{x=N-1} \sum_{y=0}^{y=N-1}(x)^{p}(y)^{q} f(x, y)
$$

where $p, q=0,1,2 \ldots$.

The moments $f(x, y)$ translated by an amount $(a, b)$ are defined as

$$
\mu_{p q}=\sum_{x} \sum_{y}(x+a)^{p}(y+b)^{q} f(x, y)
$$

\section{RESUlts}

The alphabet A in different shapes (I1, I2, I3 etc.,) has been adopted as the text image and the simulate results of average invariants $\phi_{1}, \phi_{2}, \phi_{3}$ and $\phi_{4}$ are compared with Fourier-Mellin, Legendre and Geometric Invariants as shown in table 1.

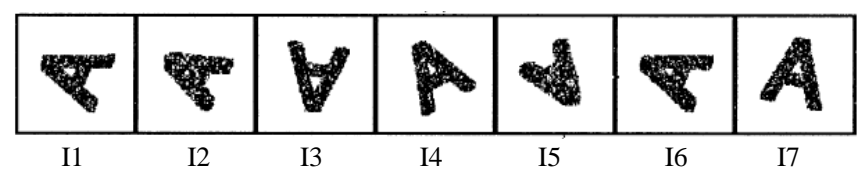

Fig. 1. Test images of the alphabet A

TABLE I: INVARIANTS FEATURES FOR COMPARISON

\begin{tabular}{cccc}
\hline Image & Fourier- Mellin & Legendre & Geometrical \\
\hline I1 & 0.16134 & 0.182431 & 0.24321 \\
I2 & 0.19169 & 0.05681 & 0.19928 \\
I3 & 0.23912 & 0.22141 & 0.26815 \\
I4 & 0.278236 & 0.18149 & 0.28816 \\
I5 & 0.284915 & 0.28551 & 0.29912 \\
I6 & 0.205873 & 0.22121 & 0.26124 \\
I7 & 0.351478 & 0.32114 & 0.368194 \\
\hline
\end{tabular}

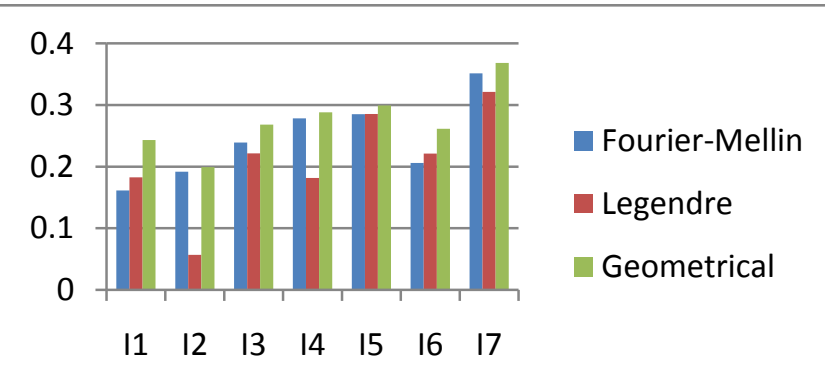

Fig.2. Performance comparison of fourier and legendre moment invariants with geometrical moment invariants

Result shows that the Geometric Moment Invariants are more superior in all aspects. The first order Invariants of Geometric Moments are compared with Fourier and Legendre Moments and are shown with bar chart.

\section{CONCLUSION}

Different Moment Invariants are compared. The first order 
Invariants of Fourier, Legendre and Geometric Moment Invariants comparison shows that Geometric Moment's Invariants are best suitable for object detection.

\section{REFERENCES}

[1] M. K. Hu, "Visual pattern recognition by moment invariants," IRE Trans Inf. Theory, vol. 8, pp. 179-187, 1962.

[2] S. A. Dudani, K. J. Breeding, and R. B. McGhee, "Aircraft identification by moment invariants," IEEE Trans. Computers, vol. 26 pp. 39-45.

[3] S. O. Belkasim, M. Shridhar, and M. Ahmadi, "Pattern recognition with moment invariants: a comparative study and new results," Pattern Recognition, vol. 24, pp. 1117-1138, 1991.

[4] Y. Li, Reforming the theory of invariant moments for pattern recognition. Patt Recog, vol. 25, pp. 723-730, 1992.

[5] W. H. Wong, W. C. Siu, and K. M. Lam. "Generation of moment invariants and their uses for character recognition," Patt Recog Lett, vol. 16, pp. 115-123, 1995.

[6] J. Flusser and T. Suk, "A moment-based approach to registration of images with affine geometric distortion," IEEE Trans. Geosciences and Remote Sensing, vol. 32, pp. 382-387, 1994.

[7] L. Wang and G. Healey, "Using Zernike moments for the illumination and geometry invariant classification of multispectral texture," IEEE Trans. Image Processing, vol. 7, pp. 196-203, 1998.

[8] T. H. Resis, "The revised fundamental theorem of moment invariants," IEEE Trans, vol. 13, pp. 830-834, 1991.

[9] M. Teague, "Image analysis via the general theory of moments," Opt. Soc. Am, vol. 70, pp. 920-930, 1980.

[10] Wallin and O. Kubler, "Complete sets of complex Zernike moment invariants and the role of the pseudo invariants," IEEE Trans. Pattern Analysis and Machine Intelligence, vol. 17, pp. 1106-1110, 1995.

[11] J. Flusser, "On the independence of rotation moment invariants," Pattern Recognition, vol. 33, pp. 1405-1410, 2000.

[12] L. V. Gool, T. Moons, and D. Ungureanu, "Affine photometric invariants for planar intensity patterns," in Proc. of 4th ECCV'96, vol. LNCS1064, pp. 642-651, Springer, 1996.

[13] J. Flusser and T. Suk, "Pattern recognition by affine moment invariants," Pattern Recognition, vol. 26, pp. 167-174, 1993.

[14] A. G. Mamistvalov, "N-Dimensional moment invariants and conceptual mathematical theory of recognition n-dimensional Solids," IEEE Trans Patt Anal Mach Intell, vol. 20, pp. 819-831, 1998.
[15] F. Ghorbel, "A complete invariant description for gray-level images by the harmonic analysis approach," Pattern Recognition Letters, vol. 15 , pp. 1043-1051, October 1994.

[16] J. Turski, "Projective Fourier analysis for patterns," Pattern Recognition, vol. 33, pp. 2033-2043, 2000.

[17] M. R. Teague, "Image analysis via the general theory of moments," J.Opt. Soc. Am., vol. 70, pp. 920-930, August 1980.

[18] C. H. Teh and R. T. Chin, "On image analysis by the method of moments," IEEE Trans. Pattern Analysis and Machine Intelligence, vol. 10, no. 4, pp. 496-513, April 1988.

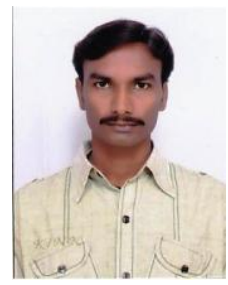

Y. Gangadhar is presently working as an Assoc. Professor in Avanthi's Scientific Technological \& Research Academy, Hyderabad-501512, A.P, India. $\mathrm{He}$ received B.Tech and $\mathrm{M}$.Tech degrees from JNTUA. He Published 5 papers in national and international journals. He Published 6 papers in national and international Conferences. His areas of interest include Digital Image Processing, Data mining, Soft Computing and Computer Networks.

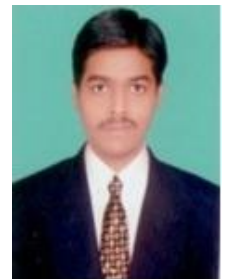

D. L. srinivasulu is presently working as an Asst. Professor in Santhiram Engineering College, Nandyal, Kurnool, A.P, India. He received B.Tech and M.Tech degrees from JNTUA. His areas of interest include Digital Image Processing and Data mining.

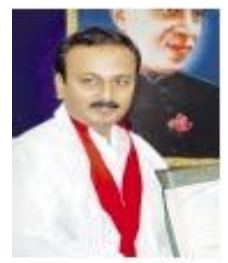

V. S. Giridhar Akula is presently working as a Professor and Principal in Avanthi's Scientific Technological \& Research Academy, Hyderabad. He received B.E, M.Tech and Ph.D degrees in Computer Science and Engineering from JNTUA. Dr Giridhar wrote 06 text books and published 22 papers in many national and international journals. He is acting as an editor and reviewer for many national and international journals. His areas of interest include Digital Image Processing, Computer Networks, Computer Graphics andArtificial Intelligence. $\mathrm{He}$ is a senior member of International Association of Computer Science and Information Technology, Singapore. 